Schneider/Ruoff/Sistermann

Umwandlungssteuer-Erlass 2011 



\title{
Umwandlungssteuer-Erlass 2011
}

\section{Kurzkommentierung mit Beraterhinweisen}

\author{
herausgegeben von \\ Dr. Norbert Schneider \\ Rechtsanwalt, Steuerberater, Dipl.-Finanzwirt (FH), Köln/Düsseldorf \\ Dr. Christian Ruoff, LLM. (Duke) \\ Rechtsanwalt, Steuerberater, Fachanwalt für Steuerrecht, \\ Attorney-at-law (New York), Hamburg
}

Dr. Christian Sistermann, LLM. International Taxation (NYU)

Rechtsanwalt, Steuerberater, München

und mit bearbeitet von

\section{Dr. David Beutel, Dr. Georg Roderburg}

Rechtsanwalt, Steuerberater, München

Rechtsanwalt, Steuerberater, Köln/Düsseldorf

Dr. Ulrich Blaas Dr. Martin Schiessl, LLM. (Exeter)

Rechtsanwalt, Steuerberater, Dipl.-Finanzwirt,

Rechtsanwalt, Fachanwalt für Steuerrecht,

Hamburg Frankfurt/Main

Dr. Jan Brinkmann, M.Jur. (Oxford) Markus Schulz

Rechtsanwalt, Steuerberater, Rechtsanwalt, Steuerberater, Dipl.-Kaufmann,

Fachanwalt für Steuerrecht, Frankfurt/Main

Dr. Benno Fischer, LLM.

Rechtsanwalt, Steuerberater, Düsseldorf

Anette Maier, LL.M.

(International Taxation, London) Köln

Dr. Alexander Schwahn, LLM.

(Stellenbosch)

Rechtsanwalt, Steuerberater, Hamburg

Ulrike Sommer

Rechtsanwältin, Steuerberaterin, Hamburg

Dr. Oliver Rode, LL.M. Dr. Tobias Teufel

Rechtsanwalt, Steuerberater, Düsseldorf Rechtsanwalt, Steuerberater, Frankfurt

bei

(92) Freshfields Bruckhaus Deringer

2012

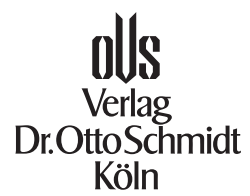




\section{Hinweis:}

In diesem Buch werden die Randnummern des Umwandlungssteuer-Erlasses 2011 als „Randnr." bezeichnet. Die Kommentierung durch die Hinweise der Autoren wird als " $\mathrm{H}^{\prime}$, gefolgt von einer zweistufigen Zählung nach Paragrafen des UmwStG und Hinweisnummer gegliedert.

\section{Zitierempfehlung:}

Ruoff in Schneider/Ruoff/Sistermann, Umwandlungssteuer-Erlass 2011, H 13.11

Bibliografische Information der Deutschen Nationalbibliothek

Die Deutsche Nationalbibliothek verzeichnet diese Publikation in der Deutschen Nationalbibliografie; detaillierte bibliografische Daten sind im Internet über http://dnb.d-nb.de abrufbar.

Verlag Dr. Otto Schmidt KG

Gustav-Heinemann-Ufer 58, 50968 Köln

Tel. 02 21/9 37 38-01, Fax 02 21/9 37 38-943

info@otto-schmidt.de

www.otto-schmidt.de

ISBN 978-3-504-24063-9

C2012 by Verlag Dr. Otto Schmidt KG, Köln

Das Werk einschließlich aller seiner Teile ist urheberrechtlich geschützt. Jede Verwertung, die nicht ausdrücklich vom Urheberrechtsgesetz zugelassen ist, bedarf der vorherigen Zustimmung des Verlages. Das gilt insbesondere für Vervielfältigungen, Bearbeitungen, Übersetzungen, Mikroverfilmungen und die Einspeicherung und Verarbeitung in elektronischen Systemen.

Das verwendete Papier ist aus chlorfrei gebleichten Rohstoffen hergestellt, holz- und säurefrei, alterungsbeständig und umweltfreundlich.

Einbandgestaltung: Jan P. Lichtenford, Mettmann

Satz: WMTP, Birkenau

Druck und Verarbeitung: Kösel, Krugzell

Printed in Germany 